\title{
Further Investigation on Building and Benchmarking A Low Power Embedded Cluster for Education
}

\author{
Sritrusta Sukaridhoto ${ }^{1}$, Achmad Subhan KHalilullah ${ }^{1}$, and Dadet Pramadihanto ${ }^{2}$
}

\begin{abstract}
Embedded parallel computing become popular, and the future of innovation in the semiconductor industry will be in ubiquitous computing. Many researchers built embedded cluster system with limited number of devices, but we utilize the device from embedded classroom to build more number of parallel computing unit. In this paper we built low power cluster consisting 32 ARM boards with low-cost customized power supply for high performance computing class for education purpose, tested with several benchmarks on embedded cluster system and analyse the raw performance.
\end{abstract}

Keywords_ Embedded Cluster System, ARM Board, Benchmark, High Computing Cluster for Education.

\section{INTRODUCTION}

$\mathrm{T}$ The large system used today in HPC are dominated by processors that use the x86 and Power instruction sets supplied by big vendors such as Intel, AMD, and IBM. These processors have been designed to mainly cater to the server, desktop PC and laptop market. The processors provide very good single thread performance but suffer from high cost and power usage. One of the main goals in building a HPC system is to stay within a power budget. In order to achieve this ambitious goal, other low power processor architectures such as embedded system are currently being explored since these processors have been primarily designed for the mobile and embedded device market.

Embedded processors (CPUs) can be found in a vast variety of products from cellular phones, digital cameras and up to network-connected household appliances. Some of these embedded processors can run advanced operating systems, such as Linux, to achieve flexible network connectivity and to have logically same functionality as that of high-end processors designed for PC and workstations. With these abilities, to build HPC system using embedded system also possible.

The issue of providing the High Performance Computing for education has been widely investigated in the literature, in particular with reference to embedded systems. In [1][2], the authors made the UCC embedded parallel computing based on SH4 processor, with 4 nodes. Sasaki, et [3], provides about M32RUCC parallel computing based on ARM processor. The node in that modules only using 4 nodes. In [4], they used Virtual Machine (VM) to learn parallel and distributed system. But still to build that system with VM it cost so much money. Balakrishnan [5] built ARM cluster but they built only less than 10 nodes and it will difficult if we use adaptor to give power supply.

One of the problems in education for HPC system is the lack of cost-effective standardized platform for prototyping, testing and evaluating application programs

\footnotetext{
${ }^{1}$ Sritrusta Sukaridhoto and Achmad Subhan KHalilullah are with Departement of Creative Multimedia, Politeknik Elektronika Negeri Surabaya, 60111, Indonesia. E-mail: dhoto@eepis-its.edu; subhankh@eepis-its.edu.

${ }^{2}$ Dadet Pramadihanto is with Departement of Information and Computer Engineering, Politeknik Elektronika Negeri Surabaya, 60111, Indonesia. E-mail: dadet@eepis-its.edu.
}

on network-connected embedded CPUs. Addressing this problem, in this paper, we present a compact high performance computing cluster system with embedded CPUs, called "EEPIS Embedded Cluster Computer (EECC)" which provides a rapid-prototyping environment for high performance computing at very low cost and low power consumption compared with conventional PC clusters.

EECC consists of 32 embedded computing nodes and network switches (100Mbps Fast Ethernet) from Embedded Class and customized power supply. The key idea is to fully utilize System on Chip (SoC) embedded products to realize cost-effective prototyping environment. In this context, we selected a commercially available embedded system as a computing node for EECC, which consists of a Dual Processor embedded CPUs, a memory, a storage, a network adapter and I/O interfaces. The computing nodes run Linux with some daemons and libraries required to make inter-processor communication for parallel processing, where MPI (message Passing Interface), and PVM (Parallel Virtual Machine) could be employed for parallel programming.

Multiple EECC can be easily stacked to extend the number of computing nodes. We decided to use System on Chip (SoC) product with embedded CPUs in building EECC. A PandaBoard ES [7] as shown in Figure 2. is employed as a computing node, is a low-power, low-cost single board computer development platform based on the Texas Instruments OMAP4460 system on a chip (SoC). The OMAP4460 SoC on the PandaBoard features a dual-core $1.2 \mathrm{GHz}$.

By the use of SoC products and customized power supply, we achieve compact size of $650 \mathrm{~mm} \times 480 \mathrm{~mm} \times$ $130 \mathrm{~mm}$ similar with $2 \mathrm{U}$ rack mount system, low power consumption of $400 \mathrm{~W}$ to drive 32 embedded CPUs and low cost. Thus, EECC can be easily introduced to educational programs in universities for Linux-based cluster computing. Another interesting feature of EECC is that every computing node has 2 USB interfaces, and hence EECC could be easily extended to various realworld application systems using USB-based sensors, such as USB cameras.

This paper is organized as follows: Section 2 gives the system overview of EECC. Section 3 describes performance test for basic data transfer bandwidth through MPI. Section 4 discusses an implementation of 
EECC as educational module in the class. In Section 5, we end with some conclusion.

\section{SYSTEM OVERVIEW}

Figure 1. shows the system overview for "EEPIS Embedded Cluster Computer (EECC)”. It consists of 16 computing nodes from \#1, \#2, until \#16, where the node $\# 1$ works as server node for various applications, such as NIS, NFS, and SSH servers, etc., and is directly accessible from terminal outside or from node \#1 which connected with monitor and keyboard. These 16 computing nodes, a network switch, and $5 \mathrm{~V}$ power supply are mounted together. The computing nodes are connected over conventional 100Mbps Fast Ethernet. Multiple EECC can be easily stacked to extend the number of computing nodes.

We decided to use System on Chip (SoC) product with embedded CPUs in building EECC. A PandaBoard ES [7] as shown in Figure 2. is employed as a computing node, is a low-power, low-cost single board computer development platform based on the Texas Instruments OMAP4460 system on a chip (SoC). The OMAP4460 SoC on the PandaBoard features a dual-core $1.2 \mathrm{GHz}$ ARM Cortex-A9 MPCore, 384 MHz PowerVR SGX540 GPU, IVA3 multimedia hardware accelerator with a programmable DSP, and 1 GB of DDR2 SDRAM. Primary persistent storage is via a SD Card slot allowing SDHC card class 10 with 8GB capacity. The board include wired 10/100 Ethernet as well as wireless Ethernet and Bluetooth connectivity. Its size is slightly larger than the ETX/XTX Computer form factor at $4 \times$ 4.5 in $(100 \times 110 \mathrm{~mm})$. The board can output video signals via DVI and HDMI interfaces. It also has $3.5 \mathrm{~mm}$ audio connectors. It has two USB host ports and one USB On-The-Go port, supporting USB 2.0. The PandaBoard has a real-time clock can be synchronize with NTP server, and runs the Linux Kernel with ARM architecture. The detailed specification of PandaBoard used for computing node is shows in Table 1.

The ARM Cortex-A9 in PandaBoard is a 32-bit multicore processor which implements the ARMv7 instruction set architecture. The cortex-A9 can have a maximum of 4 cache-coherent cores and clock frequency ranging from 800 to 2000 Mhz. Each core in the cortex-A9 CPU has a $32 \mathrm{~KB}$ instruction and a $32 \mathrm{~KB}$ data cache. One of the key features of the ARM Cortex-A series processors is the option of having Advanced SIMD (NEON) extensions. NEON is a 128-bit SIMD instruction set that accelerates applications such as multimedia, signal processing, video encode/decode, gaming, image processing etc. The features of NEON include separate register files, independent execution hardware and a comprehensive instruction set. It supports 8, 16, 32 and 64 bit integer as well as single precision 32-bit floating point SIMD operations.

EECC uses SDHC Class 10 with 8GB storage as shown in Figure 3. By using this SDHC can be easily to install Operating System such as Linux. SDHC Class 10 provides $30 \mathrm{MB} / \mathrm{s}$ transfer rate of data. With this speed gives better performance for running parallel computing inside the node.

We used DC 5V 40A 200W Transformer Switch Power Supply with cooling fan. With this power supply we can power up 16 nodes of PandaBoard. In each
PandaBoard need 5V DC with 2A minimum for requirement to run. Figure 4. Shows the type of power supply. To distribute the power supply, we made custom cables distribution board as shown in Figure 5, 6, and 7 to parallelize the supply. With this board also, we can also build stacking power supply to provide power supply for more PandaBoard.

For switching device we decided to use 24 ports 10/100Mbps. 16 ports in the switch used for PandaBoard as computing node, the rest of the port can be used as stackable network with other EECC module or can be used to connect to Internet or other LAN. Figure 8. Shows the switching device. The dimension for the switch is $28 \mathrm{~cm} \times 12.5 \mathrm{~cm} \times 4 \mathrm{~cm}$, which this size is relatively small.

The nodes run Debian GNU/Linux [8] 3.7 configured for ARMv7 on SDHC and daemons. Inside EECC there are several applications, which can support for programming, tools and parallel computing.

a. EECC supports a variety of development tools and libraries for parallel programming including GNU C, $\mathrm{C}++$ compiler, and Message Parsing Interface (MPI) by using OpenMPI [9].

b. EECC provides with a variety editor application such as Vim, nano and etc.

c. EECC also includes remote execution communication with secure shell (SSH).

d. EECC uses NFS system to share files between nodes. With midnight commander application user is able to manage file and also send to the outside of parallel computing environment.

e. EECC implements NIS to administer user accounts to be use in nodes.

f. EECC connected with EEPIS's Debian Linux local mirror to update and upgrade software.

g. With shell scripting provided by BASH, user can easily manage and control many nodes.

Figure 9. Shows a photograph of a prototype of two 16 nodes EECC modules. We could achieve extremely compact size of $650 \mathrm{~mm}$ x $480 \mathrm{~mm} \times 130 \mathrm{~mm}$ similar with $2 \mathrm{U}$ rack mount system, low power consumption of $400 \mathrm{~W}$ in total and low cost by employing the SoC embedded devices.

\section{BENCHMARK}

In this section we will discuss about the various benchmark that were run on the EECC. The benchmark system chosen based on various performance metrics of the system and cluster that is of interest to HPC applications. We describe performance of basic MPI function on EECC.

\section{A. Pallas MPI Benchmark (PMB)}

Pallas MPI Benchmark (PMB) [9] is employed to evaluate data transfer bandwidth and latency of basic MPI ping-pong, sendrecv, and exchange communications.

Figure 10 shows the result of ping-pong test, where two processors send and receive alternatively. Peak performance of ping-pong communication is estimated at about 7.5Mbytes/sec ( 60Mbps), with this result shows that EECC can communicate with enough bandwidth. Figure 11 shows the experiment result from send-recv test, where 4 processors do communication send and 
receive as full-duplex. With all processors sending and receiving packet data, EECC can utilize about 12Mbytes/sec ( 96Mbps). It means that EECC system can do high performance communication.

Figure 12 shows the result from MPI exchange communication, where 4 processors send and receive packet data as round robin topology. EECC system gave performance about 10Mbytes/sec ( 80Mbps).

\section{B. Pallas MPI Benchmark (PMB)}

High Performance Linpack (HPL) [10] is a parallel implementation of the Linpack benchmark and is portable on a wide number of machines. HPL uses double precision 64-bit arithmetic to solve a linear system of equations of order $\mathrm{N}$. It is usually run on distributed memory computers to determine the double precision floating-point performance of the system. The HPL benchmark uses LU decomposition with partial row pivoting. It uses MPI for inter-node communication and relies on various routines from BLAS and LAPACK libraries. Table 4. Shown that EECC passed the test comparing with single node in HPL test.

\section{HPC COURSE}

The course provides an introduction to advanced computer architectures, parallel algorithms, parallel languages, and performance-oriented computing, and uses real-world case studies from computational science and engineering application domains. As hardware designers turn to multi-core CPUs and GPUs, software developers must embrace parallel programming to increase performance. No single approach has yet established itself as the "right way" to develop parallel software, especially as the hardware evolves so rapidly.

We design the course that starts by overviewing the architecture of modern processors, including multi-core, many-core, and general APUs. This discussion includes not only the computation cores themselves, but also the importance of understanding the memory hierarchy and caching. The course then turns to the programmability of these systems, and works from the ground up: multithreading, higher-level directive and task-based, message-passing, and map-reduce. The course also moves from shared memory to distributed memory to the cloud, showing examples of $\mathrm{C}++11$, CUDA, Thrust, OpenMP, PPL, MPI, and Hadoop to program these systems. Additional topics include measuring performance, linear speedup, Amdahl's law, profiling and debugging tools, types of parallelism (data, task, dataflow, embarrassing), and common patterns (forkjoin, reduction, and map-reduce).

Hands-on lab exercises in $\mathrm{C}$ and $\mathrm{C}++$ are an integral part of the course; attendees should expect to bring a laptop. The syllabi of the course are as follows:

a. Intro to HPC and multicore hardware

b. Modern many-core hardware

c. Types of parallelism

d. Parallel programming in $\mathrm{C}++11$

e. The dangers of parallel programming

f. OpenMP: a higher-level abstraction

g. Task-based parallelism with the TPL

h. Tools: debuggers, profilers, and analyzers

i. Parallelism at scale: clusters and MPI

j. Parallelism at scale: cloud and Hadoop

\section{k. Future research directions}

The approach is hands-on, the students are expected to use the lecture information, a series of assignments and a final project to emerge at the end of the class with parallel programing knowledge that can be immediately applied to their research projects. The situation when students learn about HPC by using EECC system can shows in Figure 13.

In this section we compared our old HPC system with EECC system for HPC Course. The specification of our old HPC system is G4 Power Macintosh G4 (2003) as shown in Figure 14. We compared in the matter of economics, performance and education process.

Table 5 shows that comparison between old HPC systems with EECC. We give values from 1 to 10 with 1 is worst and 10 is the best value. In this comparison sometimes the bigger value is not the better meaning.

In economics way, EECC is better rather than old HPC. To build HPC system with 32 nodes using PC, we need to make investment around Rp. 160.000.000,-. But when we build HPC system using EECC it will cost around half from HPC system using PCs. The HPC system using PCs need more space comparing with EECC, because EECC size is same with $2 \mathrm{U}$ rack server. For power consumption EECC only need 400W for 32 nodes, but for PC with 550W each it will take power consumption around $17000 \mathrm{~W}$ to power up 32 nodes.

In performance way, EECC can give almost the same performance with the old HPC system. Significant different is in the matter of storage, EECC used 8 GB SDHC comparing with old HPC system used 500GB HDD. Although EECC using only 8GB storage, the education way to learn parallel computing is already enough. Basic installation of EECC system with parallel programming applications used about 2GB of storage.

In education process way, EECC gives better value rather than old HPC system. The reason is, EECC system can be used for different subject. For preparation, EECC and old HPC system are need the same action to turn on the node. We used the same material course and we added more information about embedded system. Student can learn how to make research, to find solution in HPC course.

\section{RESULT AND ANALYSIS}

In this paper, we presented a new parallel computing using embedded systems, called "EEPIS Embedded Computing Cluster (EECC)", with purpose to provide a cost-effective prototyping environment for design and test of parallel computing in education program. EECC gives a better design, low cost and also low power consumption.

For future work, we want to make EECC with better packaging and more examples for HPC course. And also we want to try EECC system in robotics system.

\section{ACKNOWLEDGEMENT}

This work is partially supported by "Penelitian Unggulan” from DIKTI 2013. The authors also want to say thank you to Mr. Nugraha Akbar and Mr. Eko Budi Utomo who helped us for designing customized power supply for this project. This research conducted at EEPIS Robotics Research Center (ER2C). 


\section{REFERENCES}

[1]. Sritrusta Sukaridhoto, Yoshifumi Sasaki, Koichi Ito, and Takafumi Aoki, "Development of a Compact Cluster with Embedded CPUs”, Proceeding of the Sixth Industrial Electronic Seminar 2004, pp.340-343, October 2004.

[2]. Yoshifumi Sasaki, Koichi Ito, Takafumi Aoki and Tatsuo Higuchi,” A compact cluster computer with embedded CPUs and its application to rapid prototyping of fingerprint verification system”, IEICE Electronics Express, Vol. 2, No.17, pp. 465-470, September 2005

[3]. Yoshifumi Sasaki, Yoshiaki Abe, "Development of an Embedded CPU Board for Cluster Computing”, SICE Tohoku branch, 2007.

[4]. Elizabeth Shoop, Richard Brown, Eric Biggers, Malcolm Kane, Devry Lin, Maura Warner, "Virtual cluster for parallel and distributed education", Proceeding of the 43rd ACM.

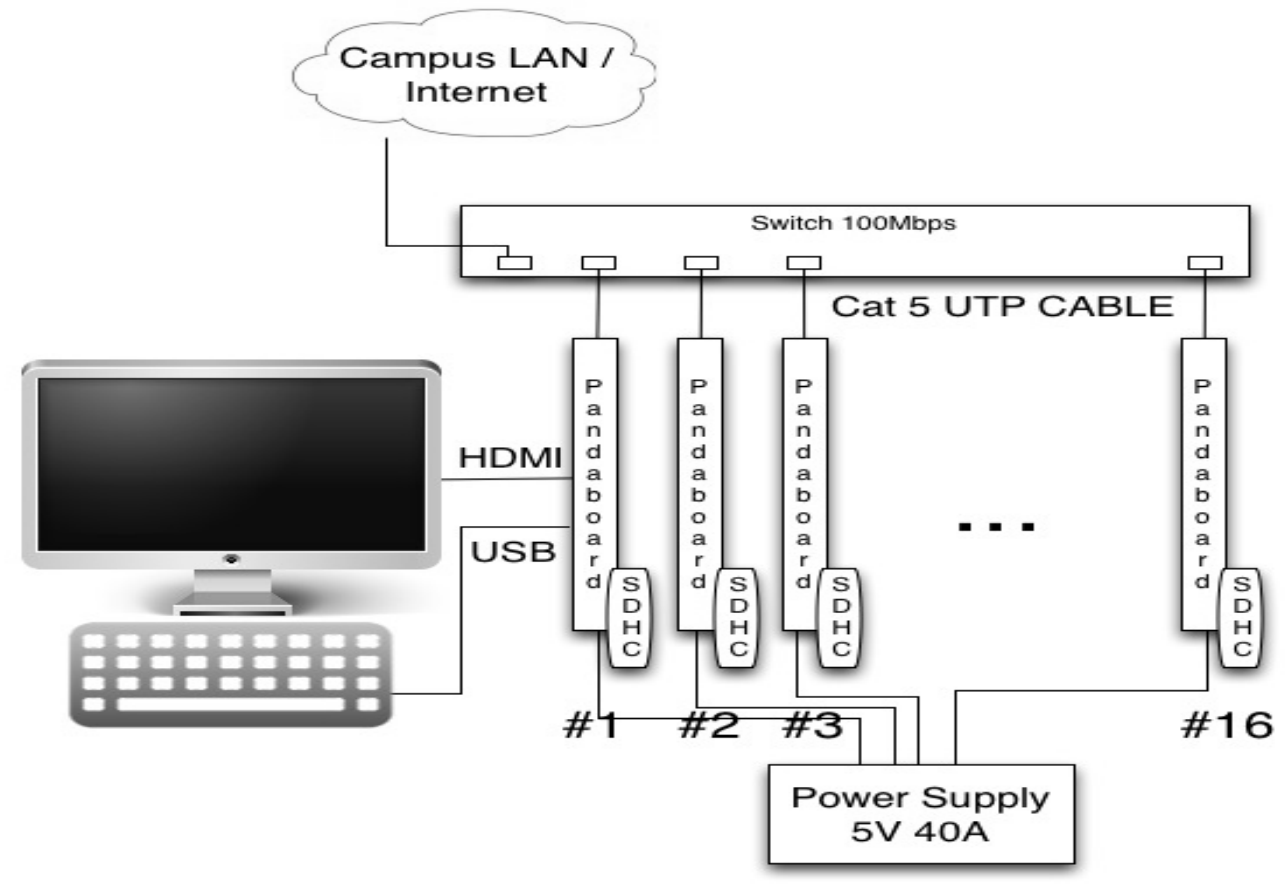

Figure 1. Architecture of the EECC

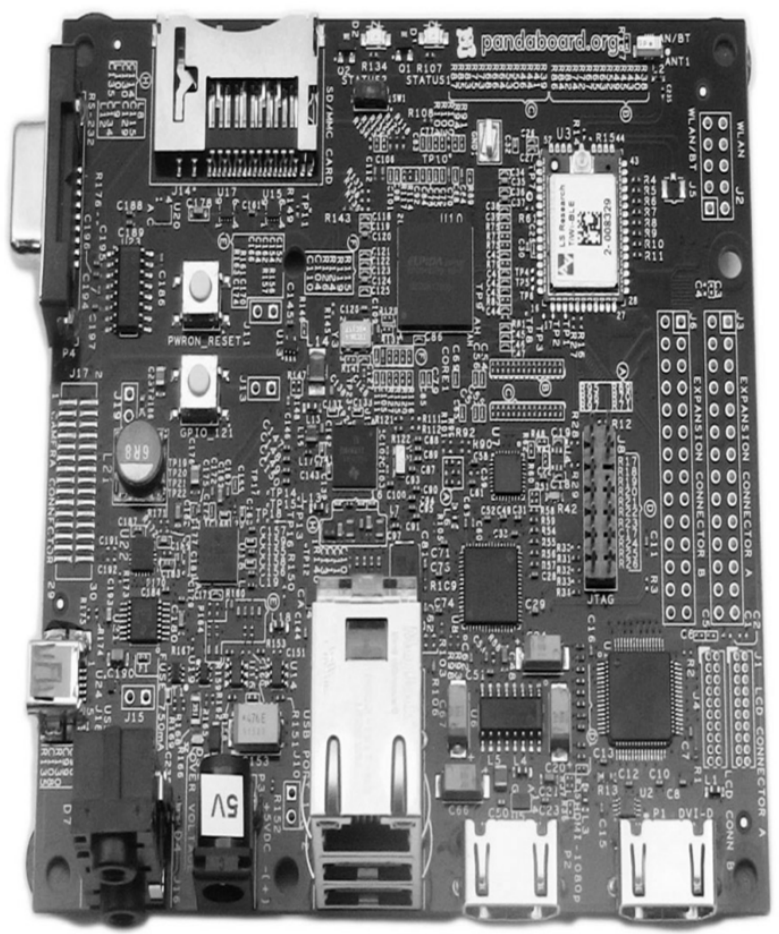

Figure 2. PandaBoard

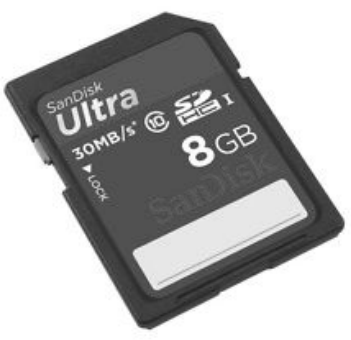

Figure 3. SDHC as Storage

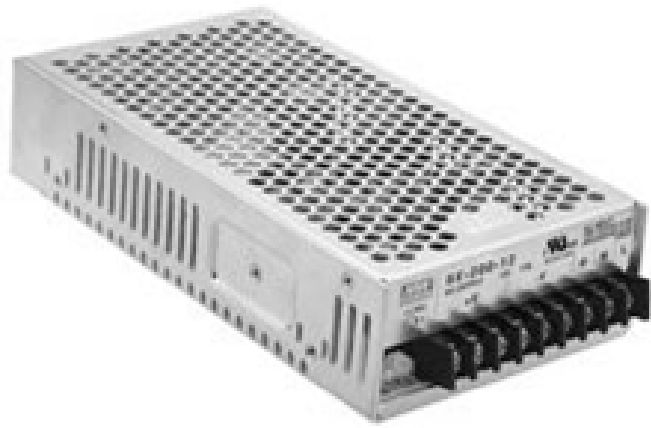

Figure 4. Power Supply DC 5V 40A to power up 16 nodes of PandaBoard 


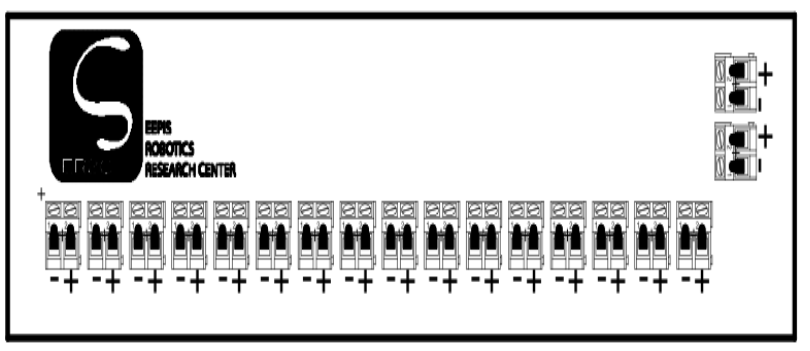

Figure 5. Top view of customized cables distribution board

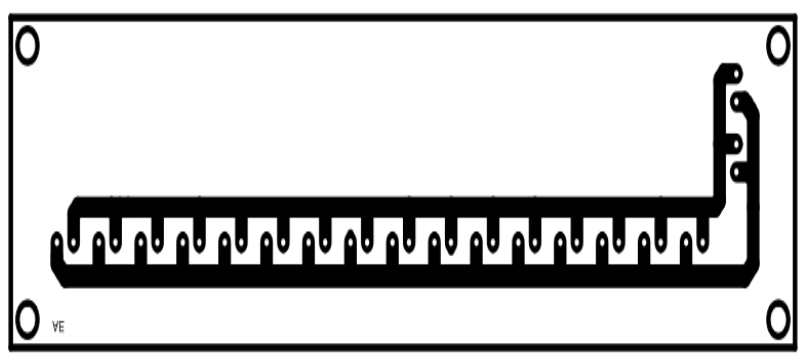

Figure 6. Bottom view of customized cables distribution board

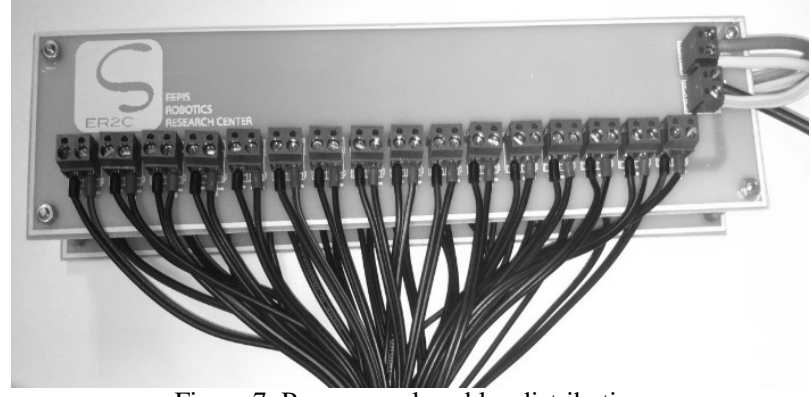

Figure 7. Power supply cables distribution

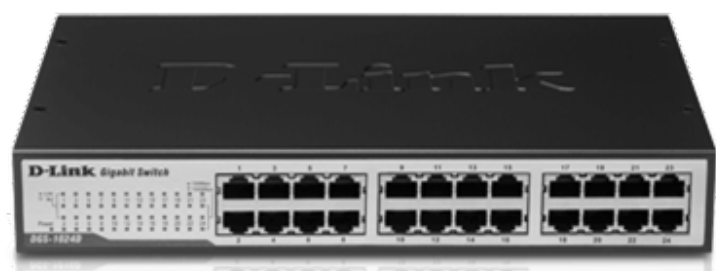

Figure 8. Switching Device

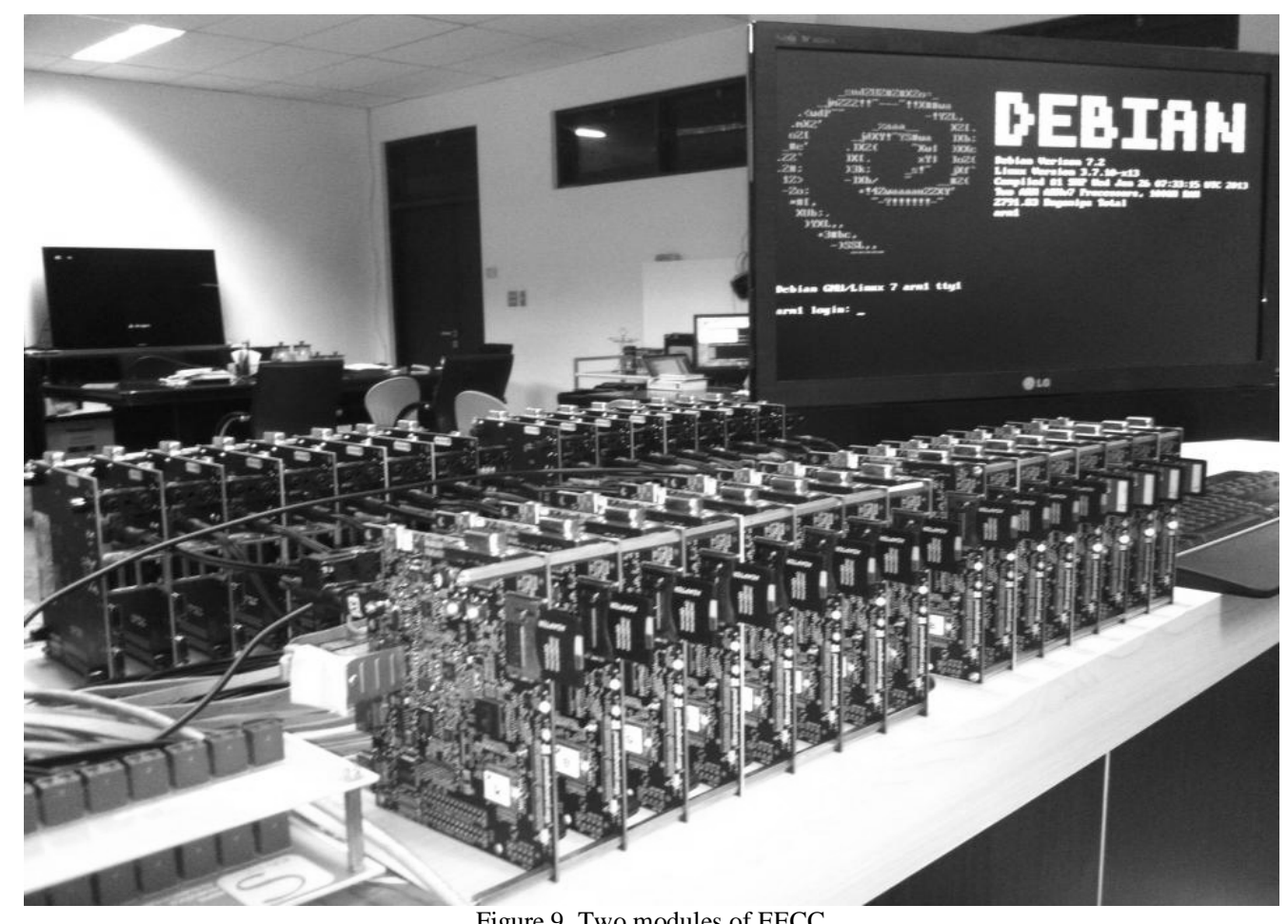

Figure 9. Two modules of EECC

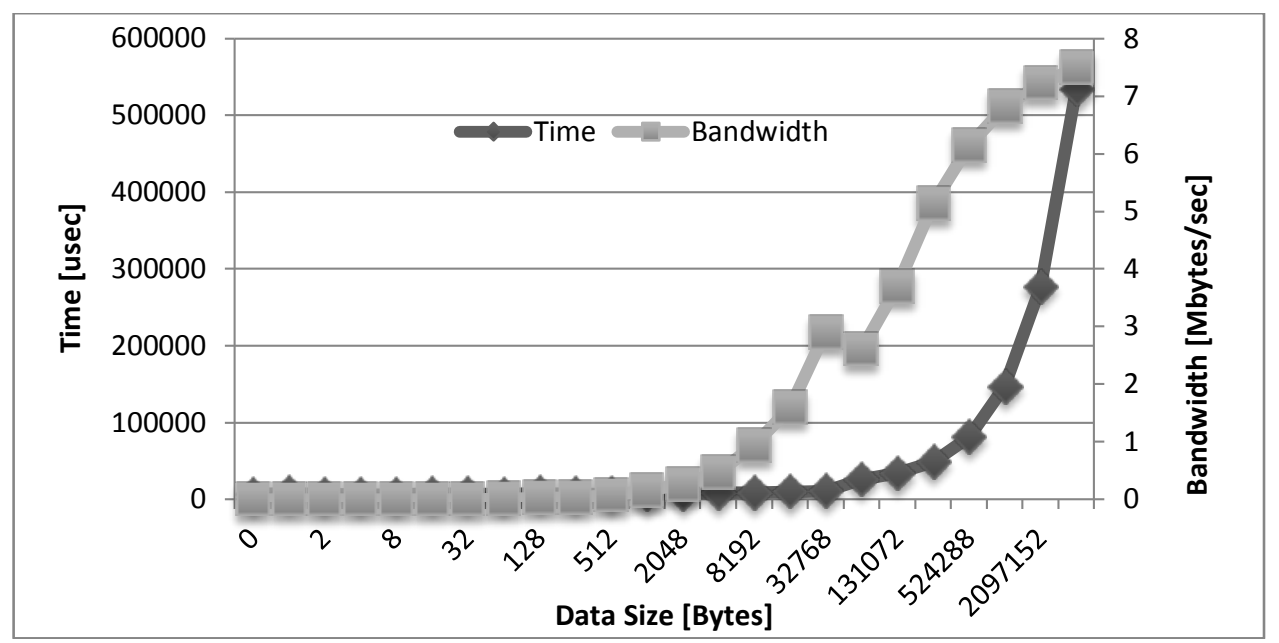

Figure 10. Benchmark for Ping-Pong 


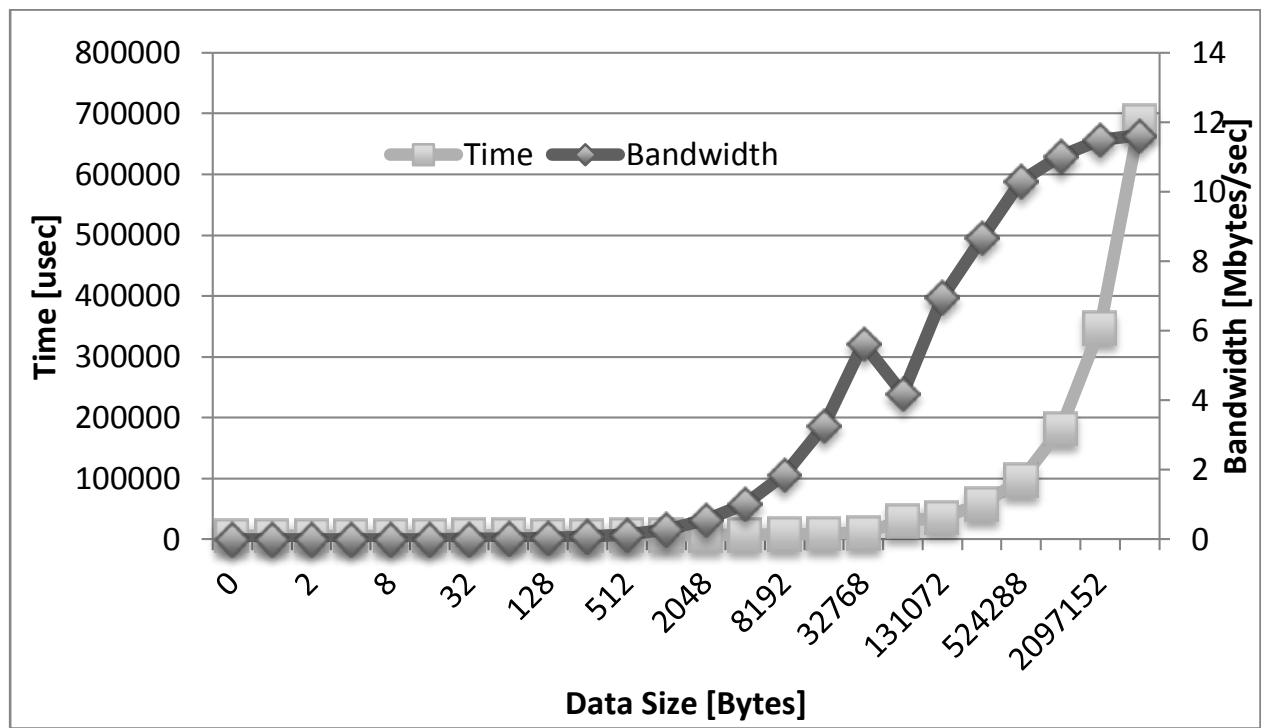

Figure 11. Benchmark for Send-Recv

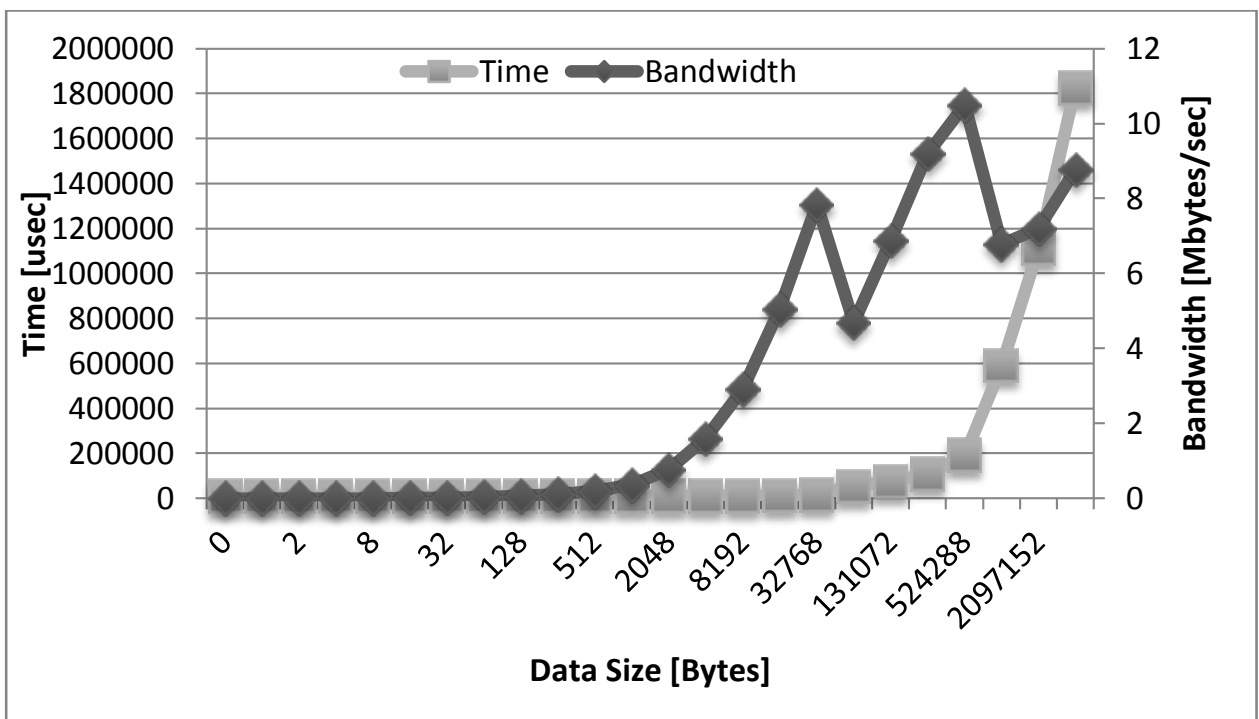

Figure 12. Benchmark for Exchange

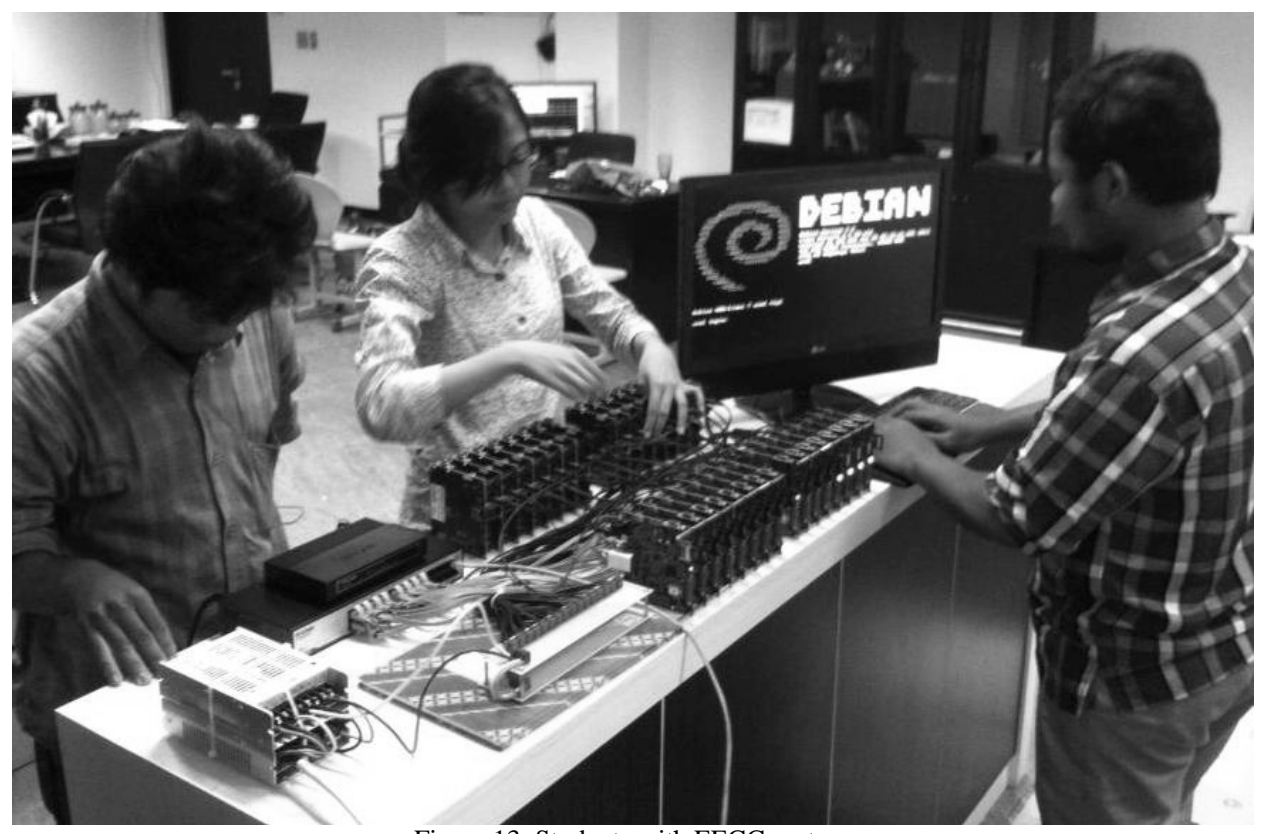

Figure 13. Students with EECC system 


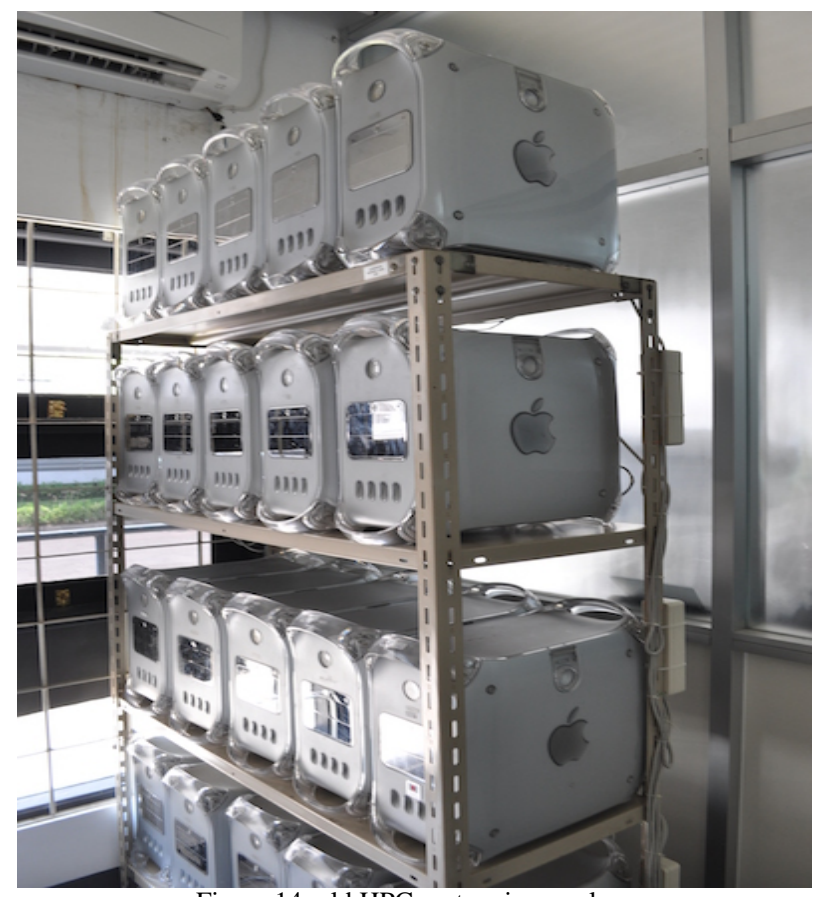

Figure 14. old HPC system in our class

TABLE 1.

PANDABOARD SPECIFICATION

\begin{tabular}{ll}
\hline \hline & PandaBoard ES \\
\hline CPU & Dual ARM Cortex-A9 MPCore \\
& $1.2 \mathrm{Ghz}$ \\
Memory & 1 GB DDR2 SDRAM \\
Storage & SDHC slot up to 32GB \\
Network & 100Mbps Ethernet \\
& 802.11n Wifi \\
& Bluetooth \\
Interface & DVI \\
& HDMI \\
& 2x USB 2.0 \\
& $1 x$ USB On-The-Go \\
Graphics & SGX540 GPU \\
Audio & 3.5 mm \\
Size & 4 x 4.5 in \\
Weight & 81.5 gr \\
Power Supply & DC 5V, Min 2A $\sim$ Max 4A \\
Clock & Real-time \\
OS & Debian Linux 3.7 armhf \\
\hline \hline
\end{tabular}

TABLE 2.

POWER SUPPLY SPECIFICATION.

\begin{tabular}{lll}
\hline & Model & PS200-W1V \\
\hline Output & DC Voltage & $5 \mathrm{~V}$ \\
& Current Range & $0 \sim 40 \mathrm{~A}$ \\
& Rated Power & $200 \mathrm{~W}$ \\
Input & AC Current & $2.5 \mathrm{~A} / 230 \mathrm{VAC}$ \\
& Voltage range & $170 \sim 260 \mathrm{VAC}$ \\
& Efficiency & $81 \%$ \\
\hline
\end{tabular}

TABLE 3.

OVERALL SPECIFICATION OF EECC.

\begin{tabular}{|c|c|c|}
\hline \multicolumn{2}{|c|}{ Employed computing nodes } & SoC PandaBoard ES \\
\hline \multicolumn{2}{|c|}{ Number of computing nodes } & 16 \\
\hline \multicolumn{2}{|c|}{ Network interface } & 100Mbps Fast Ethernet \\
\hline \multicolumn{2}{|c|}{ Power consumption } & 160W 200W \\
\hline \multicolumn{2}{|l|}{ Size (mm) } & $650 \times 240 \times 130$ \\
\hline \multirow[t]{6}{*}{ Software } & Operating system & $\begin{array}{l}\text { Debian GNU Linux } 3.7 \\
\text { armhf }\end{array}$ \\
\hline & Server function & NIS NFS \\
\hline & Communication & SSH \\
\hline & Development & GNU C, C++ \\
\hline & & Vim \\
\hline & & OpenMPI \\
\hline
\end{tabular}


TABLE 4.

HPL RESULTS

\begin{tabular}{ccccc}
\hline \hline Test & Single & \multicolumn{2}{c}{ MPI } & max \\
\hline & & min & avg & 0.010214 \\
\hline Random Access (GUP/s) & 0.009839 & 0.005963 & 0.009027 & 0.609617 \\
DGEMM (Gflop/s) & 0.602819 & 0.48892 & 0.560694 & 0.672081 \\
STREAM copy (GB/s) & 0.672 & 0.661977 & 0.664542 & 0.672404 \\
STREAM Scale (GB/s) & 0.672404 & 0.662056 & 0.669736 & 0.704745 \\
STREAM Add (GB/s) & 0.662029 & 0.655306 & 0.658719 & 0.704745 \\
\hline STREAM Triad (GB/s & 0.697131 & 0.682608 & 0.693598 & \\
\hline \hline
\end{tabular}

TABLE 5.

COMPARISON OF OLD HPC SYSTEM WITH EECC

\begin{tabular}{|c|c|c|c|c|}
\hline No & Statement & $\begin{array}{l}\text { HPC with } \\
\text { PC/Mac }\end{array}$ & EECC & explaination \\
\hline \multicolumn{5}{|c|}{ Economics } \\
\hline 1 & Cost & 10 & 5 & $\begin{array}{l}\text { To build HPC system with PC/Mac it cost @Rp. 5.000.000, but EECC } \\
\text { only @2.500.000 }\end{array}$ \\
\hline 2 & Space & 10 & 2 & $\begin{array}{l}\text { EECC size is same with } 2 \mathrm{U} \text { rack server smaller then PC } \\
\text { PC power consumption more than } 550 \mathrm{~W} / \mathrm{PC} \text {, but EECC is around }\end{array}$ \\
\hline 3 & Power consumption & 10 & 1 & 400W for 32 nodes \\
\hline 4 & Device replacement & 10 & 10 & $\begin{array}{l}\text { PC waiting time for new device is longer rather than EECC } \\
\text { In EECC we can unplug the SDHC, reinstall. But PC need to prepare }\end{array}$ \\
\hline 5 & Easy to install & 5 & 10 & storage and other stuff \\
\hline 6 & Number of nodes & 5 & 10 & EECC are 32 nodes, PC are 16 nodes \\
\hline
\end{tabular}

\section{Performance}

$\begin{array}{llccl}1 & \text { Parallel mechanism } & 10 & 10 & \text { Can do parallel computing using MPI, PVM etc } \\ 2 & \text { Speedup factor } & 10 & 9 & \text { Our PC specification is almost the same with PandaBoard } \\ 3 & \text { Networking } & 10 & 10 & \text { Run on 100Mbps } \\ 4 & \text { Storage } & 10 & 8 & \text { PC have 500GB, EECC have 8GB } \\ 5 & \text { Interface } & 8 & 9 & \text { EECC also have HDMI and Wireless communication, not PC }\end{array}$

\section{Education process}

\begin{tabular}{llccl}
1 & Preparation & 8 & 9 & Turn on the system \\
2 & Teaching material & 10 & 10 & use the same material \\
3 & Student knowleadge & 9 & 9 & student can understand the same way \\
4 & Long-life education & 9 & 9 & student can find the solution if they found some problems \\
5 & Research & 7 & 9 & with embedded system we can try different field of research \\
6 & Exam & 8 & 8 & Student can do the task or exam \\
7 & Use devices for & 4 & 9 & HPC from PC is dedicated only for HPC subject, while EECC can be \\
\hline \hline
\end{tabular}

